Proceedings of the 2011 Winter Simulation Conference

S. Jain, R.R. Creasey, J. Himmelspach, K.P. White, and M. Fu, eds.

\title{
ENHANCING OPERATIONAL EFFICIENCY OF A CONTAINER OPERATOR: A SIMULATION OPTIMIZATION APPROACH
}

\author{
Santanu Sinha \\ Viswanath Kumar Ganesan \\ Complex Decision Support Systems \\ Tata Consultancy Services Ltd. \\ Mumbai 400093, INDIA
}

\begin{abstract}
One of the key issues in a typical marine logistics industry dealing with container operations is to maximize profitability subject to pre-specified service level compliance(s) under uncertain and complex business environment. The problem becomes more complex in presence of heterogeneous customers, varied degree of demand priority, supply restrictions, and other allied operational constraints. In this paper, a typical container business operation has been considered where the service provider deals with different types of customers. The problem has been modeled with discrete-event simulation techniques. A simulation optimization technique has been deployed to analyze several opportunities to improve overall system performance in terms of increased profit, demand fulfillment rate, and deriving other contractual parameter(s) under varied scenarios. Trade-offs between different KPIs including fleet size, unmet demand, service level, and utilization have been analyzed and a sensitivity analysis has been provided to bring in several managerial insights.
\end{abstract}

\section{INTRODUCTION}

Globalization of world economy has accelerated the use of containers in cargo transportation through Third Party Logistics Providers (3PL) and today over 60\% of the world's maritime cargo is transported in containers, while in some routes among the economically strong countries the figure has gone up to $100 \%$ (Steenken, Vob, and Stahlbock 2004). As an effective mode of merchandise transportation, use of containers not only has reduced the handling and transportation costs, but also has been considered as a faster and more efficient delivery option to thousands of customers in a geographically distributed network (Bandeira, Becker, and Borenstein 2009). Issues related to container operations have gained significant attention and have been extensively studied under various modeling contexts. The entire gamut of research can be captured into two dimensions - conceptual framework and solution technology.

In the perspective of conceptual framework, the related models can broadly be categorized into twocost minimization and profit maximization. In the former, some authors have focused on cost minimization models which deal with designing efficient reposition plan, forecasting, safety-stock determination, and tactical fleet planning problem (Erera, Morales, and Savelsbergh 2005; Li et al. 2007). An excellent review has been provided by Powell (2005). The other direction considers the revenue maximization aspects based on heterogeneous customer attributes. This stream of research merges the concept of both revenue management through dynamic pricing and cost minimization through efficiency enhancement. Not many of the revenue maximization models have been developed for container industry.

In the perspective of solution technology, a stream of research is based on mathematical programming where the authors have developed optimization models and solution algorithms under different modeling assumptions (White 1972, Turnquist and Jordan 1986). In all the cases, the authors have focused on de- 


\section{Sinha and Ganesan}

veloping mathematical models to manage dynamic fleet operations. Specific to container industry, several crucial research efforts have been carried out (Crainic, Gendreau, and Dejax 1993; Erera, Morales, and Savelsberg 2005). Further, a general model for managing dynamic fleets under various transportation modes has been described by Powell (2005). Another stream of research on container operations is based on computer simulation. In this sense, computer simulation has been deployed to address several business issues such as, improving the performance of container terminal operations (Yun and Choi 1999), liner shipping operations (McLean and Biles 2008), empty container repositioning (Jula, Chassiakos, and Ioannou 2006), and the problem of determining optimal fleet size under various operational constraints (Donga and Song 2009).

From the review of literature, it has been observed that though traditional mathematical and optimization techniques have been successfully applied to solve diverse business problems in the domain of container operations, these techniques cannot capture system behavior in an uncertain and dynamic environment where there are several interactions among the system components (Sage and Rouse 2009). In a typical 3PL business model, there are several parameters with significant variability and uncertainties such as, customer arrival rate, customer demand, full-move transit time, empty reposition time, capacity allocation, and reposition policy. The complexity goes even higher in presence of heterogeneous customers with varied degree of contribution/profitability and service expectations. In such situations, simulation is considered to be an important and efficient tool to analyze system variability and randomness and capture system behavior under various conditions through several what-if scenarios.

Thus, we have been motivated to address the issues of container operations with segmented demand. In this effort, we merge both the aspects of revenue maximization and cost minimization together in a basic 3PL business model with two different types of customers, viz., high and low priority. A simulation-based approach has been deployed to analyze the system behavior over the planning horizon and determine the optimal control parameters. We have investigated the trade-off between segmented demand and service-level and the issue of determining optimal fleet size corresponding to target demand fulfillment rate for any type of customers.

Further, we analyze a typical practice of delayed delivery which is very common in container/3PL industry where customer is asked to wait for sometime till the containers/equipments are available. A simulation optimization technique has been applied to determine the optimal length of such waiting time considering other factors such as overall demand fill rate, revenue, profit, and service level compliance.

The remainder of the paper is organized as follows. The problem of container business operation has been briefly discussed in section 2. A simulation model has been developed in section 3. In section 4, we briefly discuss about simulation optimization. We carry out several simulation experiments and elaborate the results in section 5. Finally, we summarize and conclude in section 6.

\section{PROBLEM DESCRIPTION}

In this paper, the concepts of revenue management are evaluated for a container operator (a typical 3PL) that transports materials for its customers from one location to another in a geographically dispersed supply chain network. The 3PL operation consists of the following functions:

1. Management of customer orders for empty containers

2. Serving customers by dispatching empty containers to the customer locations subject to the availability of containers at the respective service depots

3. Loading of materials and shipment of containers from customer locations to destinations

4. Reposition of empty containers back to the appropriate service depot(s) to serve future customer demand therein

Though the 3PL service provider operates on hundreds of unique origin-destination pair, for the purpose of illustration and tractability, without losing generality, we assume three major hubs or zones of operation by aggregation and $3 \times 3=9$ trade-lanes as shown in Figure 1 where, $d_{i}=$ demand at $i, v_{i j}, c_{i j}$ are 


\section{Sinha and Ganesan}

revenue and cost respectively between trade-lane $i$ to $j$. Here, $\lambda_{i}\left[d_{i}\right]$ represents the stochastic arrival rate $\lambda_{i}$ of Customers along with stochastic demand $\widetilde{d}_{i}$ at location $i(i=1,2,3)$.

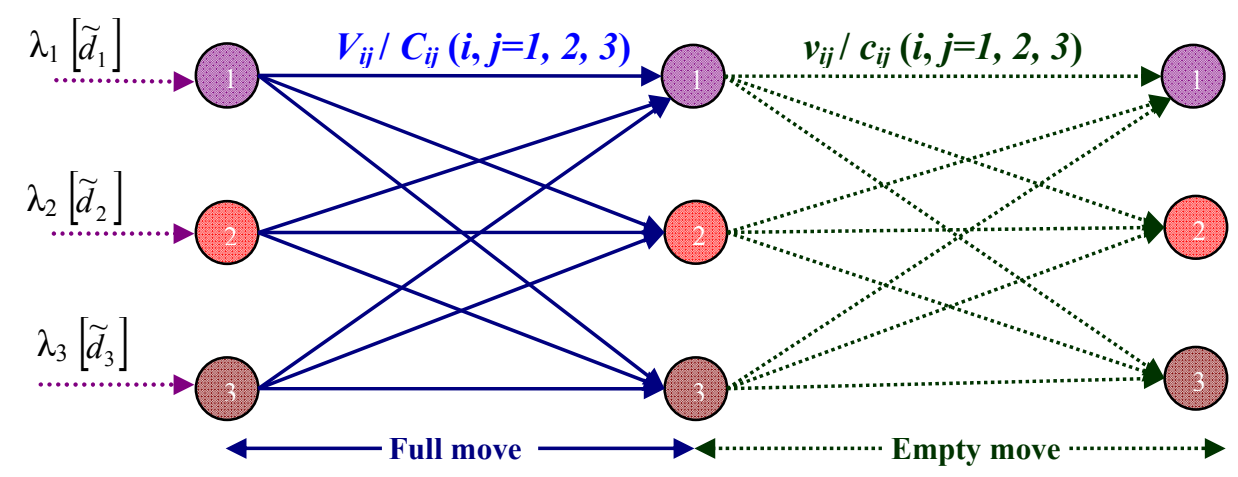

Figure 1: Container business operation model with $3 \mathrm{X} 3$ trade-lanes

For simplicity of exposition, we consider that the operator manages a homogeneous fleet of containers. We further consider that the service provider faces two different types of customers - high priority and low priority - depending on their marginal yield per capacity. The order fulfillment happens on a first-in-first-out (FIFO) basis; but in case of a capacity shortage, we assume that the high priority customers are given higher priority governed by a contractual agreement $\{W T, P\}$ which guarantees a delayed order fulfillment within an additional $W T$ days - failing which the service provider is subjected to pay a penalty of $P$ per ordered capacity to the respective high priority customer. The overall order fulfillment can be represented as shown in Figure 2. In this case, the issue is to determine the optimal WT corresponding to a specific penalty charge $P$ under dynamic and uncertain business environment. Further, trade-offs between different KPIs (fleet size, unmet demand, service level, utilization) have been analyzed and a sensitivity analysis has been provided to bring in several managerial insights.

\section{BUSINESS MODEL OVERVIEW}

The inputs, business metrics, and the control variables used for modeling the 3PL process operations are elaborated in this section.

\subsection{Input Parameters}

The following input parameters (factors) have been considered in the business model $(i, j=1,2,3)$ :

- Customer arrival rate in location $i$

- Percentage $\left(p_{i}\right)$ of high-priority customers in location $i$

- Average demand for containers in location $i$

- $\quad$ Percentage of shipment between $i$ to $j$

- Revenue per container in trade lane $i$ to $j$, for high priority customer

- Revenue per container in trade lane $i$ to $j$ for low priority customers

- Transit time from $i$ to $j$ for full load container

- Logistics cost to move a single container from $i$ to $j$

- Reposition time from $i$ to $j$ for empty load container

- Reposition cost per container from $i$ to $j$. 


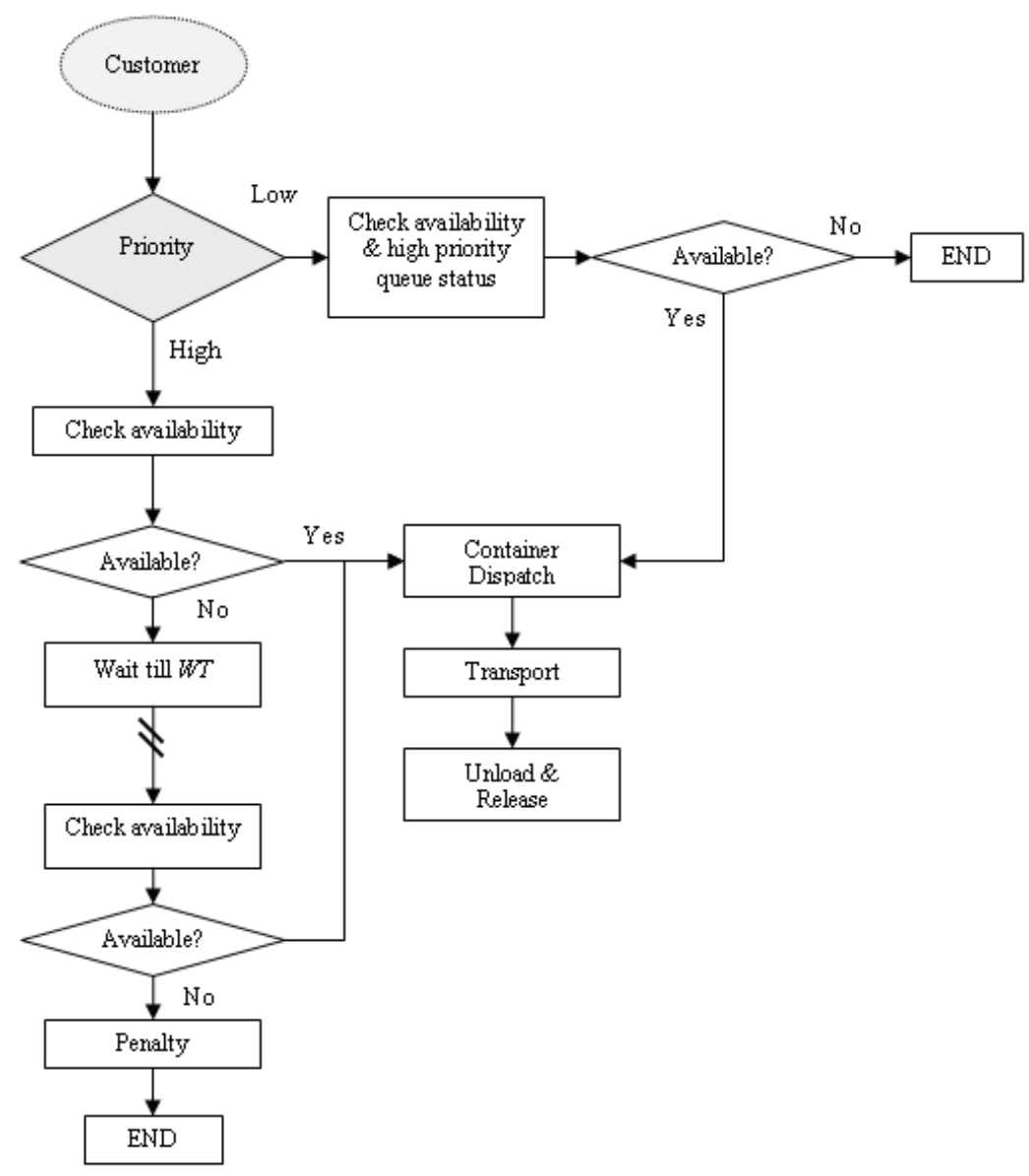

Figure 2: Order fulfillment process with high and low priority customers

\subsection{Business Metrics}

The following parameters (responses) have been considered to measure the performance of the business model:

- Gross profit

- Operating cost

- Revenue

- Unmet demand (UMD)

- Container utilization

\subsection{Control Variables}

The following control variables having a direct influence on any of the above mentioned business objective, have been considered in the model:

- Service level $\left(S L_{k}\right)$, where $k=1,2$ indicating the high and low priority customers, respectively

- Reposition policy

- Contractual waiting time for premium customers - in case of inventory shortage (WT)

- Fleet size $(F S)$

Safety-stock level at location $i$ has been calculated as follows: 


$$
S S_{i}=\sum_{k=1}^{2} S L_{k} \sqrt{L T_{i}\left(\sigma_{d k}\right)_{i}^{2}+\left(D_{k}\right)_{i}^{2}\left(\sigma_{L T}\right)_{i}^{2}}
$$

where, $L T_{i}$ and $\left(\sigma_{L T}\right)_{i}$ represent the average and standard deviation of supply lead-time while $D_{k_{i}}$ and $\left(\sigma_{d k}\right)_{i}$ indicate the average and standard deviation of demand for customer type $k$ at location $i$. Thus, if the safety-stock level at a location $i$ falls below the expected level $S S_{i}$, the reposition policy ensures the earliest replenishment of empty containers from other sources having excess capacity.

Further, we assume that the contractual back order lead time (WT), i.e., the waiting time of the high priority customers to be served in case of a capacity shortage, is a variable and can have significant impact on the overall performance of the system. With simulation optimization, we have shown how an optimal $W T$ can be derived for a specific fleet size (FS), and other input parameters. We also include a sensitivity study with the various input parameters. The container utilization is calculated as follows:

$$
\text { Utilization }=\frac{\sum(\text { total containers that served customers }) .(\text { corresponding transit t ime })}{\text { (Simulation time }) \cdot(F S)}
$$

The objective is to measure the system performance with the help of the following KPIs: gross profit, operating cost, revenue, unmet demand, and utilization. The typical questions that could help improving business performance are:

- What should be the optimal $W T$ for a specific fleet size (FS), and other input parameters to minimize unmet demand or maximize revenue?

- What are the trade-offs between fleet size and other key performance indicators (KPIs)?

With all the complexities mentioned above, the proposed model seems to be complex and mathematically intractable. In such situations, simulation is an important and popular tool that considers variability and randomness in the business processes and captures the system behavior under various conditions by analyzing several what-if scenarios.

\section{SIMULATION OPTIMIZATION}

Recently, with the increasing power of computing devices, simulation optimization has become an effective way to merge the benefits of both simulation and optimization to find a set of model specifications (i.e., input parameters and/or structural assumptions) that leads to optimal performance (April et al. 2005). More specifically, simulation optimization is an optimization technique where the performance is measured on the basis of the output of a simulation model. The problem settings contains the usual optimization components, such as, decision variables $(x)$, objective function $f(x)$, and the associated constraints $(Q)$ (Ólafsson and Kim 2002). The objective function is estimated using a function of the stochastic simulation output say, $g(x)$ defined in a real space, i.e., $f: Q \rightarrow R$. The objective function typically might be an unbiased estimate of the true objective function, that is, $f(x)=E[g(x)]$. More details are available in Ólafsson and Kim (2002), April et al. (2003), and Fu, Glover, and April (2005). Accordingly, in simulation optimization, the optimization engine chooses a set of values for the controllable inputs and these are used as inputs to the simulation engine. Based on the performance of the simulation output, the optimizer selects the next trial solution until the termination criteria is achieved or a desired level of improved output is obtained. A detailed review of various techniques has been provided by Azadivar (1999). A typical example of such application in a 3PL industry has been illustrated in Sinha and Ganesan (2009). 


\section{Sinha and Ganesan}

In this paper, the various aspects of operational and tactical planning in the scope of the above mentioned model are evaluated with simulation optimization and several insights have been provided in scope of managerial decision making under diverse contexts.

\section{SIMULATION EXPERIMENTS AND RESULTS}

Three different business cases have been considered herein to provide various insights on application of revenue management principles and operations planning with simulation optimization techniques. A popular simulation tool ARENA 10 (refer ARENA Simulation Software; Source: www.arenasimulation.com/Products_OptQuest.aspx) has been deployed to model, simulate, and optimize the business operation process discussed above.

\subsection{Base Case}

This case has been used as a reference to compare the benefits of implementing Revenue Management (RM) practices using simulation and optimization engines. The base case considers uniform customer arrival rate and dispatch volume across all lanes. Reposition of empty containers is assumed to follow the simple rule:

"If current inventory at location $i$ is more than the desired safety stock $S_{i}$ AND (Current Inventory + Planned receipt) at location $j$ is less than safety stock $S S_{j}$, Then Move empty containers from location i to $j . "$

For any location $i$, the safety stock level $S_{i}$ can be estimated from (1) where the service level for both type of customers have been considered as $80 \%$, i.e. $S L_{1}=S L_{2}=0.80$. Further, for the fleet size of 150 initially distributed uniformly, the trade-off between back order time and the corresponding profit can be shown in Table 1.

Table I: Results of the base case simulation $\left(p_{i}=0.5\right)$

\begin{tabular}{lllllll}
\hline Sr. & WT (hr) & Profit & Overall SL & $\mathrm{SL}_{1}$ & $\mathrm{SL}_{2}$ & Utilization \\
\hline 1 & 0 & 507490.0 & $79.5 \%$ & $79.5 \%$ & $79.5 \%$ & $39.0 \%$ \\
2 & 25 & 529495.0 & $80.7 \%$ & $87.0 \%$ & $74.6 \%$ & $39.7 \%$ \\
3 & 50 & 534440.0 & $80.7 \%$ & $92.2 \%$ & $70.3 \%$ & $39.8 \%$ \\
4 & 60 & 540685.0 & $82.8 \%$ & $95.2 \%$ & $70.5 \%$ & $39.8 \%$ \\
5 & 75 & 537270.0 & $81.6 \%$ & $95.0 \%$ & $69.6 \%$ & $39.8 \%$ \\
6 & 100 & 556410.0 & $81.7 \%$ & $97.3 \%$ & $66.2 \%$ & $40.5 \%$ \\
7 & 125 & 535230.0 & $82.2 \%$ & $99.1 \%$ & $66.0 \%$ & $38.8 \%$ \\
8 & 150 & 537675.0 & $84.0 \%$ & $99.8 \%$ & $68.8 \%$ & $39.3 \%$ \\
9 & 200 & 548645.0 & $81.4 \%$ & $99.8 \%$ & $63.7 \%$ & $39.7 \%$ \\
10 & 250 & 549485.0 & $82.7 \%$ & $100.0 \%$ & $66.2 \%$ & $40.2 \%$ \\
11 & 300 & 549485.0 & $82.7 \%$ & $100.0 \%$ & $66.2 \%$ & $40.1 \%$ \\
\hline
\end{tabular}

This shows that the maximum profit is achieved with $W T=100$. However, it is tedious to generate large number of scenarios to find out the optimal $W T^{*}$. The next model establishes the benefit of simulation optimization in such cases to derive optimal parameter value. 


\section{Sinha and Ganesan}

\subsection{Optimization of Base Case Model}

The earlier base case model has been optimized with simulation optimization with OptQuest embedded in ARENA. The optimization engine deploys various algorithms based on Tabu search, scatter search, integer programming, and neural networks to improve performance objectives.

In this case, the back order time WT has been considered as a variable and the optimal $W T^{*}$ has been found at 90.05 hrs. The variation of net profit with back order duration $(W T)$ is shown in Figure 3.

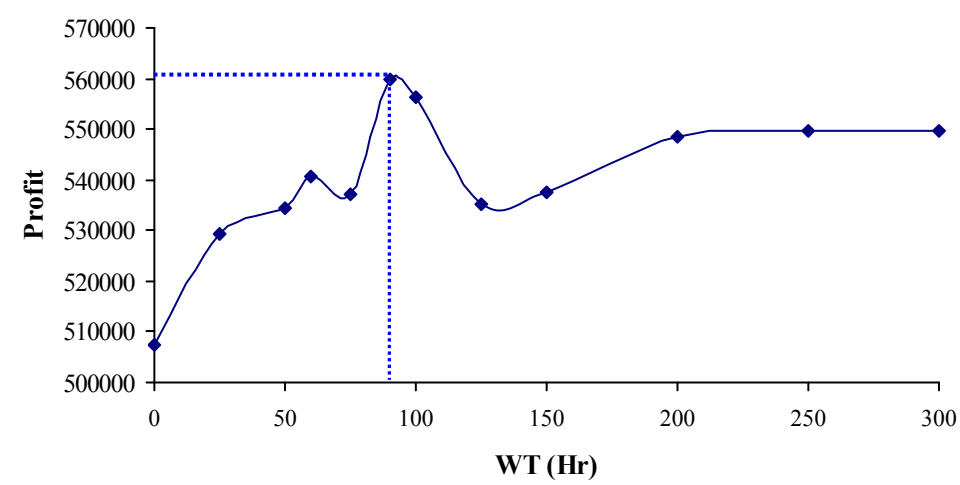

Figure 3: Optimization of base case model

This shows a further $0.65 \%$ increase of profit - as compared to the earlier case. The impact of the optimal WT on service levels on two different types of customers has been shown in Figure 4.

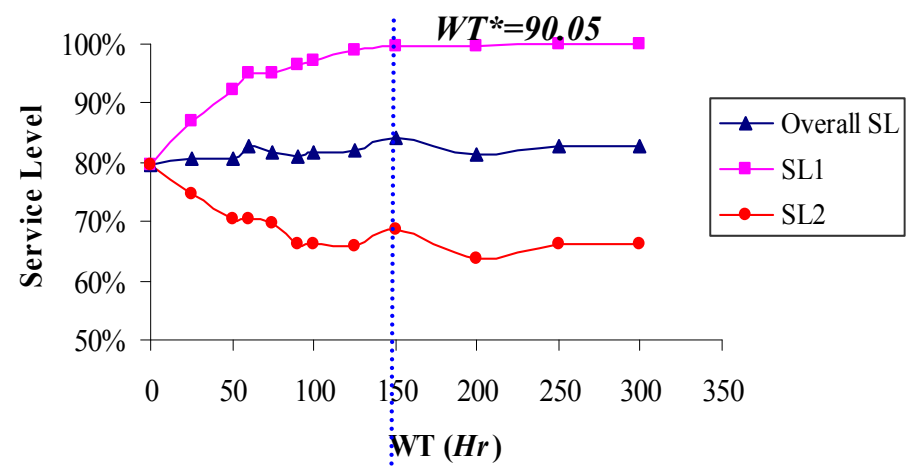

Figure 4: WT vs. Service level

\subsection{Base Case Model with Optimization under Varied Proportions of Customer Types}

Next, we derive the optimal $W T$ under varied proportion $p_{i}$ of high-priority customers in location $i$. The results are tabulated in Table 2.

\subsection{Capacity Planning}

The demand fulfillment rate for high priority customers drops at increased service levels. This is attributed to the fact that higher safety stock level results in less free containers. However, demand fulfillment rate for low priority customers increases at increased service levels - this is due to the fact that additional fleet base increases the chance of possible fulfillment of low priority orders - after serving the high priority ones. The impact of fleet sizes and services levels across the two types of customers is demonstrated in 


\section{Sinha and Ganesan}

the following figures. Figure 5a and 5b demonstrates the performance at $100 \%$ SL and $85 \%$ SL for high and low priority customers respectively when serving different proportions of demand among two types of customers.

Table 2: Optimal $W T^{*}{ }^{*}$ vs. $p_{i}$

\begin{tabular}{lllllll}
\hline$p_{i}$ & Optimal $W T$ & Profit & Overall $S L$ & $S L_{1}$ & $S L_{2}$ & Utilization \\
\hline $0 \%$ & -- & 359305 & $79 \%$ & -- & $79 \%$ & $39.0 \%$ \\
$10 \%$ & 59.25 & 404520 & $78 \%$ & $98 \%$ & $76 \%$ & $39.3 \%$ \\
$25 \%$ & 115.55 & 474120 & $80 \%$ & $99 \%$ & $72 \%$ & $39.9 \%$ \\
$50 \%$ & 90.03 & 560025 & $81 \%$ & $100 \%$ & $64 \%$ & $40.7 \%$ \\
$75 \%$ & 463.80 & 694410 & $86 \%$ & $99 \%$ & $44 \%$ & $43.5 \%$ \\
$100 \%$ & 413.95 & 799585 & $95 \%$ & $95 \%$ & -- & $47.2 \%$ \\
\hline
\end{tabular}

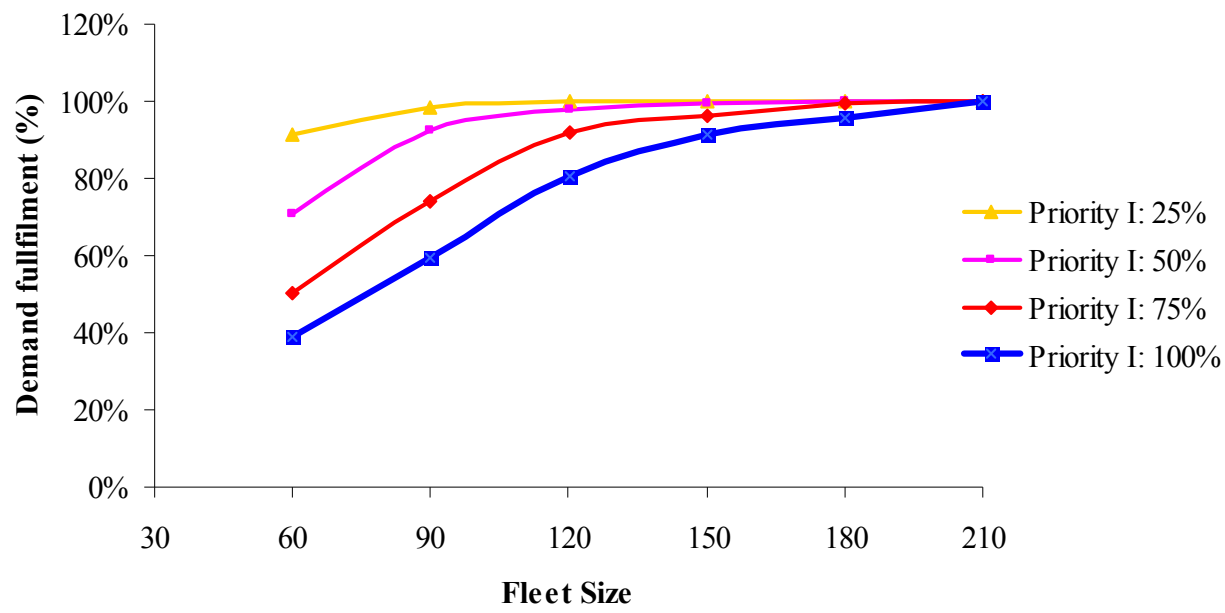

Figure 5a: Fleet size vs. demand fulfillment (P1 Customers)

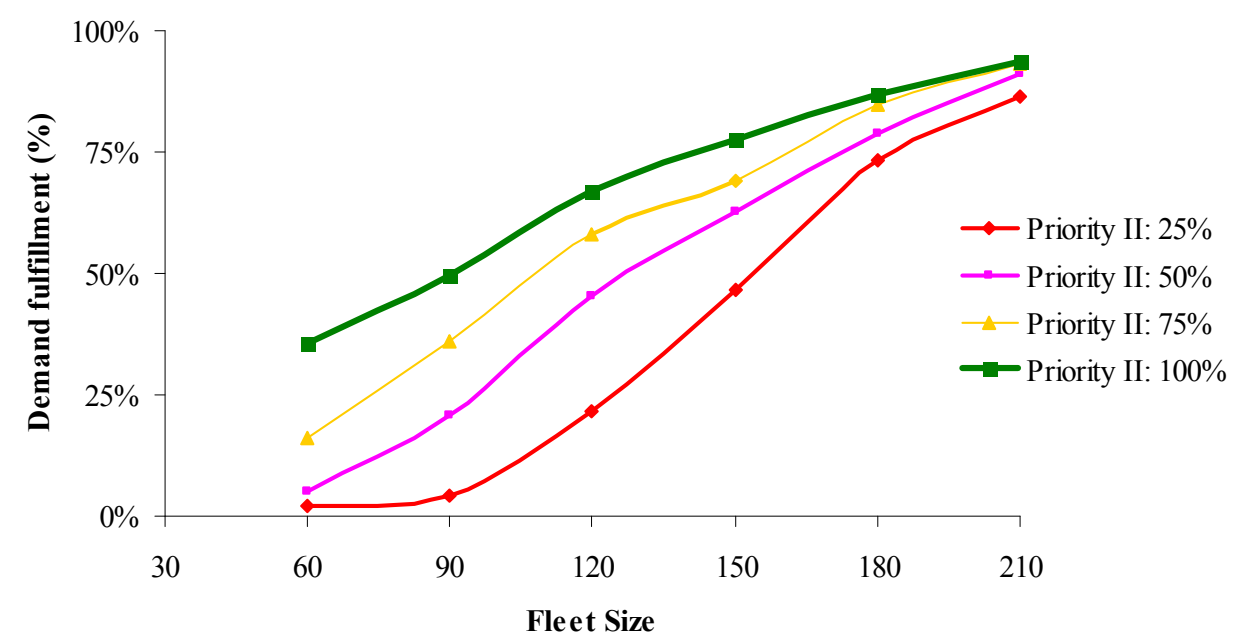

Figure 5b: Fleet size vs. demand fulfillment (P2 Customers) 


\section{Sinha and Ganesan}

Deriving optimal fleet size: Figure $5 \mathrm{c}$ demonstrates how to arrive at an optimal fleet size corresponding to given Service Levels and expected demand fulfillment rate. As an example, in order to meet $90 \%$ and $70 \%$ service level for high and low priority customers respectively and to maintain an aggregate $92 \%$ demand fulfillment rate, the optimal fleet size is 120 .

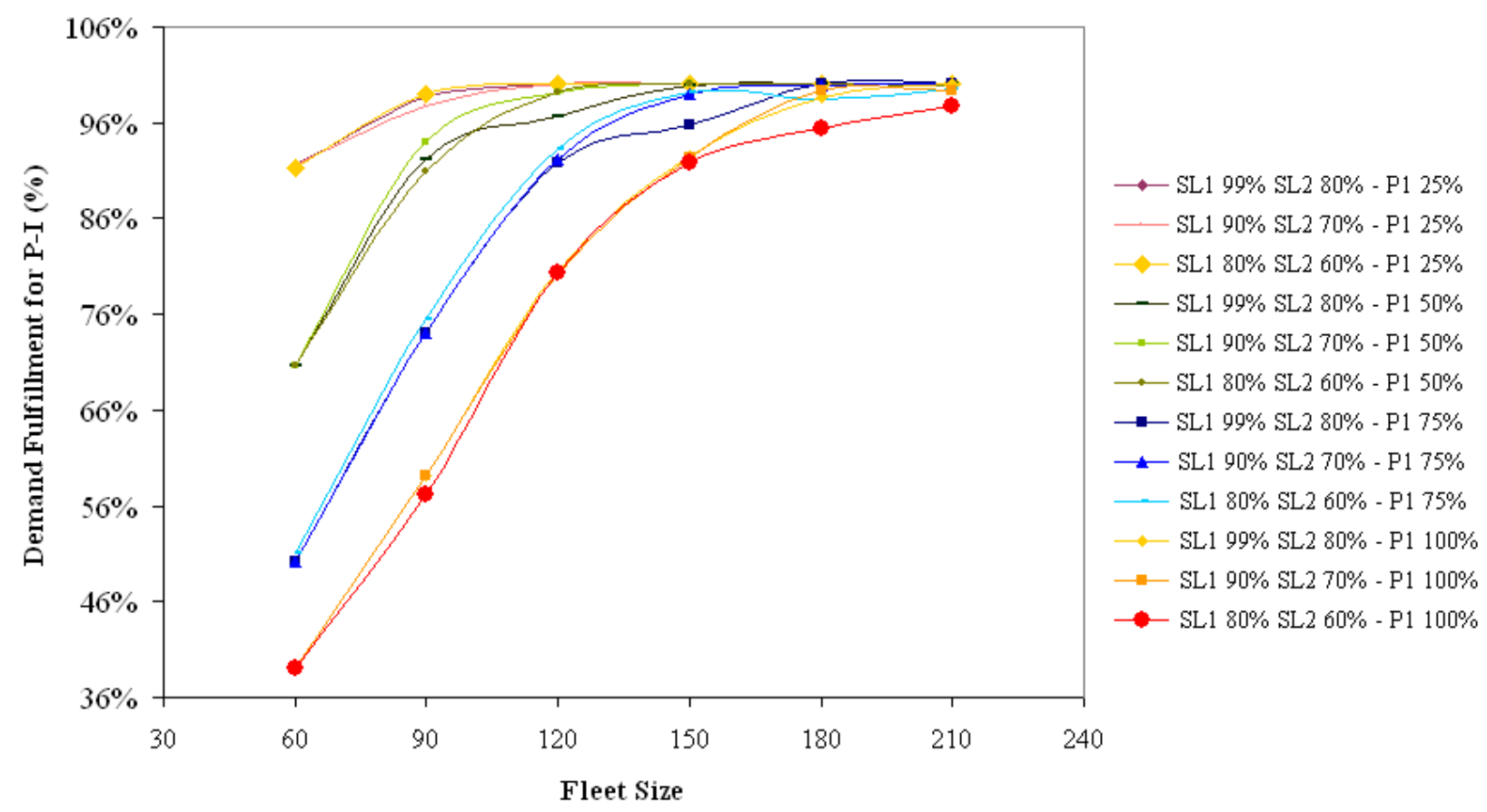

Figure 5c: Fleet size vs. demand fulfillment

Fleet size vs. Net Profit: Following the experiments, it has been found that net profit increases with increase in $\mathrm{P}_{1}$ type of customers - because of higher contribution. However, though higher fleet size initially results in higher profit, further increase reduces the net profit due to high capital expenditure. This observation is quite evident from figure 6 .

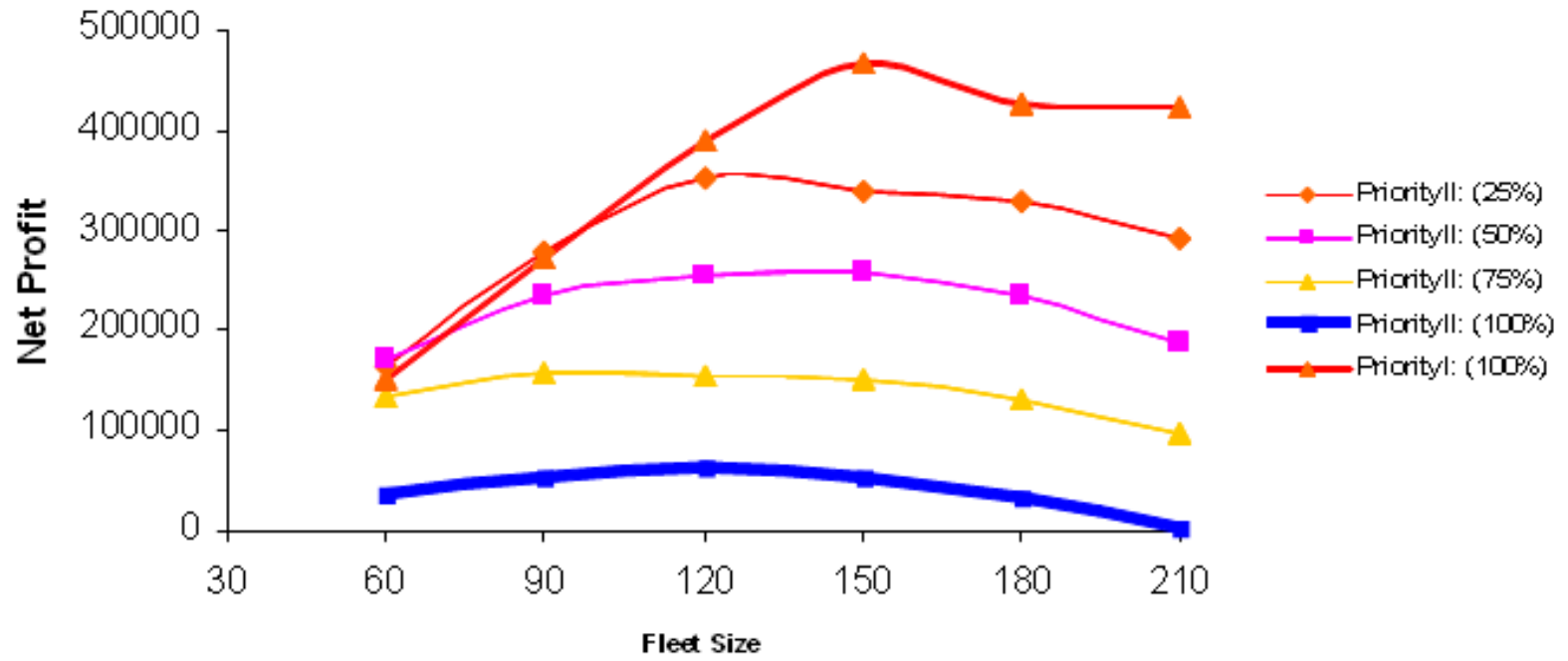

Figure 6: Fleet size vs. net profit 


\section{Sinha and Ganesan}

Sensitivity analysis: The following figure shows the trade-off between Fleet size (FS), Service level (SL) and net profit. The following variations have been considered:

- Large FS: 210 Containers

- Low FS: 60 Containers

- High SL: SL1=99\%, SL2=80\%

- Low SL: SL1=80\%, SL2=60\%.

The results for P1 Customer demand are shown in Fig. 7. The results for P2 Customer demand has been found to be a mirror image of Fig. 7 due to the fact that P1 and P2 demand always equal to $100 \%$ when added.

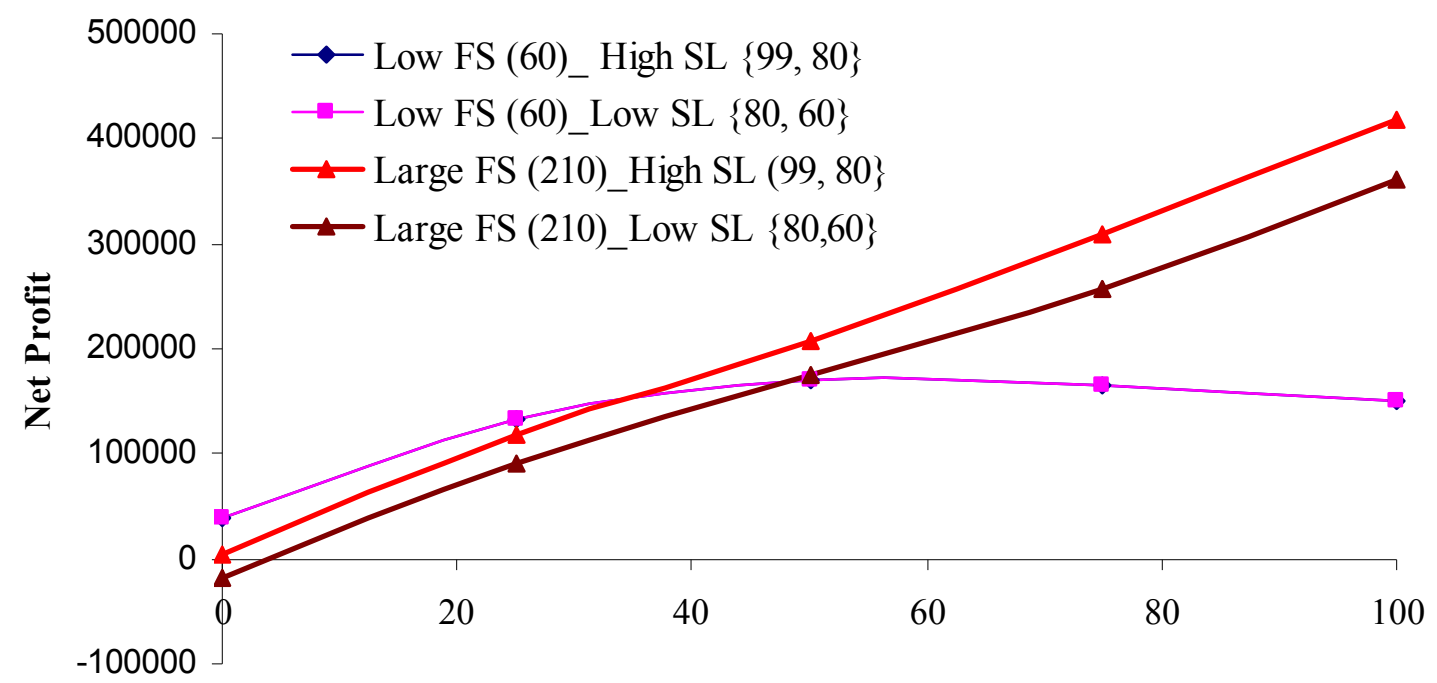

P-I customer demand $(\%)$

Figure 7: Fleet size, net profit and P1 customer demand

It has been observed that service level does not have much impact on net profit if fleet size is low - as due to small fleet size, maintaining the safety stock level becomes difficult due to capacity shortage. Other observations related to the system behavior are as follows:

For a given fleet size: As $p_{1}$ increases, gross revenue increases, simultaneously unmet demand for priority customer (UMD-I) increases with higher penalty cost, Thus, net profit (NP) decreases. However, if $p_{1}$ increases beyond a certain level, depending on the marginal revenue and unit penalty charge, additional penalty charge may be offset by additional revenue leading to increased net profit.

For larger fleet size: With larger fleet size, UMD-1 decreases. As a result, penalty decreases. But because of higher cost related to procure additional fleets (Capex), net profit decreases. However, if $p_{1}$ increases gross revenue also increases. Thus, sometimes, the additional Capex is offset by additional revenue. In such cases, net profit increases.

If fleet size is small and safety stock (SS) is high, containers remain idle at some places and capacity utilization decreases. However, if fleet size increases up to a certain level, utilization increases because of more free/available containers - beyond which utilization again starts decreasing due to high safety stock levels or surplus capacity. 


\section{Sinha and Ganesan}

\section{CONCLUSION}

Managing container business operations with heterogeneous customer demand and service priority under uncertain and complex business environment has been a key challenge for the practitioners. In order to address the issue, this paper has studied a typical container operation process and applied discrete-event simulation technique to improve overall system performance in terms of increased profit, demand fulfillment rate, and deriving other contractual parameter(s) under varied scenarios. The problem has been modeled in the perspective of revenue management practices assuming different types of customers with varied levels of priority to improve profitability and service level agreement.

The proposed model has shown how simulation and optimization can be applied to decide the optimal order/service lead time for the high priority customers to maximize profit. Further experiments have been conducted to provide several insights on system behavior with input parameters such as, fleet size, service level, and proportion of high priority customers. Impact of such input parameters on the key performance indicators such as, demand fulfillment rate, profit, utilization, and operating cost, has also been discussed following a sensitivity study.

It has finally been shown how simulation and optimization can help taking more informed decisions to significantly improve the overall performance level of a container business process and also help in taking various strategic decisions such as fleet size and service level agreements under diverse business environments.

\section{ACKNOWLEDGMENT}

We sincerely acknowledge Dr. Siddhartha SenGupta, Head, Complex Decision Support Systems (CDSS) group for his invaluable inputs and constant guidance throughout the work. We are also thankful to Dr. Sumit Raut, Dr. Sudhir Sinha, Dr. Sharadha Ramanan, and Mr. Shripad Salsingikar, Tata Consultancy Services, for their critical comments and helpful suggestions during the research and preparation of the earlier manuscripts.

\section{REFERENCES}

April, J., F. Glover, J. Kelly, and M. Laguna. 2003. "Practical Introduction To Simulation Optimization." In Proceedings of 2003 Winter Simulation Conference, edited by S. E. Chick, P. J. Sanchez, D. M. Ferrin, and D. J. Morrice, 71-78. Piscataway, New Jersey: Institute of Electrical and Electronics Engineers, Inc.

April, J., M. Better, F. Glover, J. P. Kelly, and M. Laguna. 2005. "Enhancing Business Process Management with Simulation Optimization.” BP Trends January 2005, Accessed March 15. http://www.bptrends.com/publicationfiles/01-05\%20WP\%20Simulation\%20Optimization\%20\%20April\%20et\%20al.pdf.

Azadivar, F. 1999. "Simulation Optimization Methodologies." In Proceedings of the 1999 Winter Simulation Conference, edited by P.A. Farrington, H.B. Nembhard, D.T. Sturrock, and G.W. Evans, 93-100. Piscataway, New Jersey: Institute of Electrical and Electronics Engineers, Inc.

Bandeira, D. L., J. L. Becker, and D. Borenstein. "A DSS for Integrated Distribution of Empty and Full Containers." Decision Support Systems 47: 383-397.

Crainic, T. G., M. Gendreau, and P. Dejax. 1993. "Dynamic and Stochastic Models for the Allocation of Empty Containers." Operations Research 41(1): 102-126.

Donga, J., and D. Song. 2009. "Container Fleet Sizing and Empty Repositioning in Liner Shipping Systems." Transportation Research Part E: Logistics and Transportation Review 45(6): 860-877.

Erera, A. L., J. C. Morales, and M. Savelsbergh. 2005. "Global Intermodal Tank Container Management for the Chemical Industry." Transportation Research Part E: Logistics and Transportation Review 41: 551-566. 
Fu M. C., F. W. Glover, and J. April. 2005. "Simulation optimization: A review, new developments, and applications", In Proceedings of the 2005 Winter Simulation Conference, edited by M.E. Kuhl, N. M. Steiger, F. B. Armstrong \& J. A. Joines, 83-95. Piscataway, New Jersey: Institute of Electrical and Electronics Engineers, Inc.

Jula, H., A. Chassiakos, and P. Ioannou. 2006. "Port Dynamic Empty Container Reuse." Transportation Research Part E: Logistics and Transportation Review 42(1): 43-60.

Li, J-A., S. C. H. Leung, Y. Wu, and K. Liu, 2007. "Allocation of empty containers between multi-ports." European Journal of Operational Research 182(1): 400-412.

McLean, A. A., and W.E. Biles. 2008. "A Simulation Approach to the Evaluation of Operational Costs and Performance in Liner Shipping Operations", In Proceedings of the 2008 Winter Simulation Conference, edited by S. J. Mason, R. R. Hill, L. Mönch, O.Rose, T. Jefferson, and J. W. Fowler, 257785. Piscataway, New Jersey: Institute of Electrical and Electronics Engineers, Inc.

Ólafsson S., and J. Kim. 2002. "Simulation Optimization." In Proceedings of the 2002 Winter Simulation Conference, edited by E. Yücesan, C. H. Chen, J. L. Snowdon, J. M. Charnes, 79-84. Piscataway, New Jersey: Institute of Electrical and Electronics Engineers, Inc.

Powell, W. B. 2005. "Dynamic Models of Transportation Operations." In Supply Chain Management: Design, Coordination and Operation, Handbooks in Operations Research and Management Science, 11: 677-7. Elsevier.

Sage, A. P., and W. B. Rouse. 2009. Handbook of Systems Engineering and Management. John Wiley \& Sons, Inc.

Sinha S. and Ganesan V. 2009. "Dynamic Modelling of Business Process Flows and Transformations using Simulation Optimization." In Proceedings of the $7^{\text {th }}$ AIMS International Conference on Management, 1440-50. IIM Bangalore, India. December, 2009.

Steenken, D., S. Vob, and R. Stahlbock. 2004. "Container Terminal Operation and Operations Research: a Classification and Literature Review." OR Spectrum 26: 3-49.

Turnquist, M. A., and W. C. Jordan. 1986. "Fleet Sizing Under Production Cycles and Uncertain Travel Times." Transportation Science 20(4): 227-236.

White, W. 1972. "Dynamic Transshipment Networks: An Algorithm and its Application to the Distribution of Empty Containers." Networks 2(3): 211-236.

Yun, W. Y., and Y. S. Choi. 1999. "A Simulation Model for Container-Terminal Operation Analysis using an Object Oriented Approach.” International Journal of Production Economics 59: 221-230.

\section{AUTHOR BIOGRAPHIES}

SANTANU SINHA obtained his Ph.D from the Indian Institute of Technology Kharagpur, India and is currently working as Assistant Consultant in the applied research group "Complex Decision Support Systems" at Tata Consultancy Services Ltd., Mumbai. His current research interests are in the areas of supply chain management, logistics, inventory management and optimization. The author has already published papers in international journals including, Mathematical and Computer Modeling, Computers \& Industrial Engineering, International Journal of Operational Research, etc. His email address is <santanu.sinha@tcs.com>.

VISWANATH KUMAR GANESAN obtained his Ph.D from the Indian Institute of Technology Madras, India and is currently working as Consultant in the applied research group "Complex Decision Support Systems" at Tata Consultancy Services Ltd., Mumbai. His current research interests are in the areas of supply chain management, logistics, inventory management and optimization. The author has already published papers in international journals including, European Journal of Operational Research, Computers \& Industrial Engineering, Computers \& Operations Research, International Journal of Production Economics, etc. His email address is <viswanath.ganesan@tcs.com>. 